\title{
Measuring access to urban health services using Geographical Information System (GIS): a case study of health service management in Bandar Abbas, Iran
}

\author{
Mehdi Masoodi ${ }^{1}$, Mahsa Rahimzadeh ${ }^{2,3^{*}}$
}

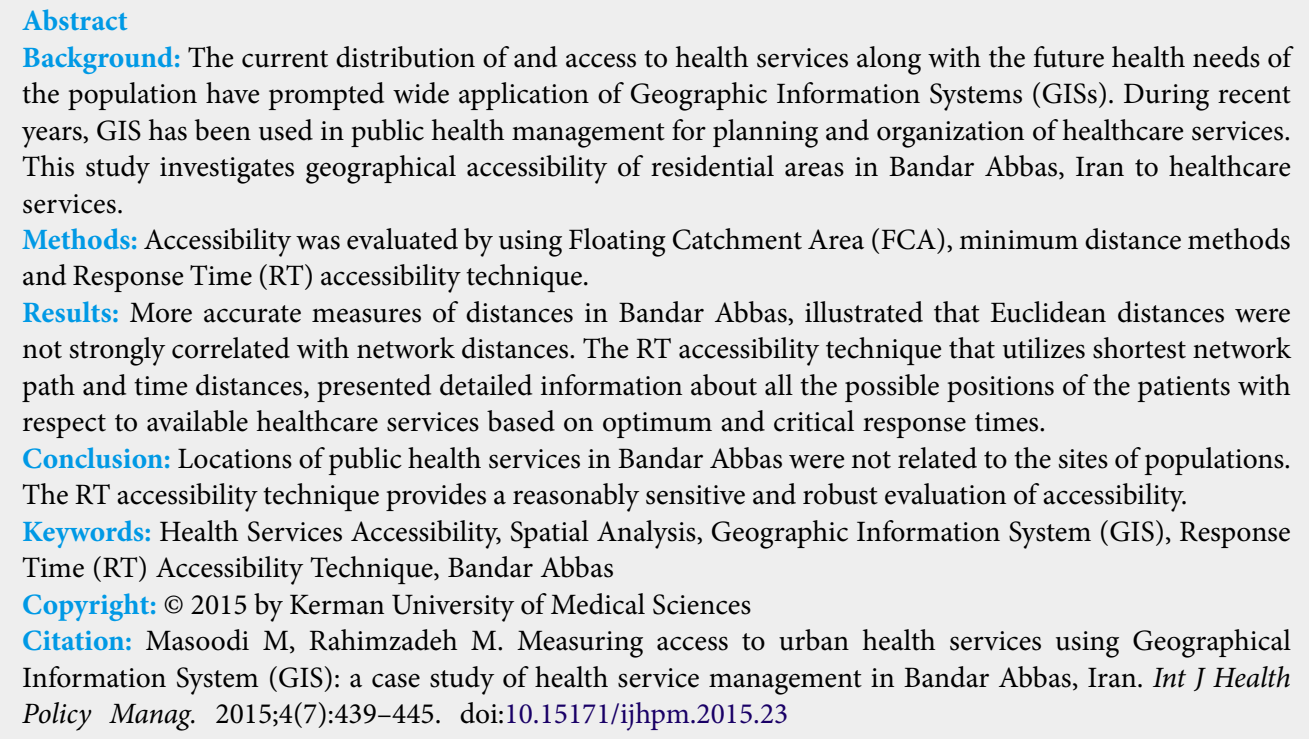

Background: The current distribution of and access to health services along with the future health needs of the population have prompted wide application of Geographic Information Systems (GISs). During recent years, GIS has been used in public health management for planning and organization of healthcare services. This study investigates geographical accessibility of residential areas in Bandar Abbas, Iran to healthcare services.

Methods: Accessibility was evaluated by using Floating Catchment Area (FCA), minimum distance methods and Response Time (RT) accessibility technique.

Results: More accurate measures of distances in Bandar Abbas, illustrated that Euclidean distances were not strongly correlated with network distances. The RT accessibility technique that utilizes shortest network path and time distances, presented detailed information about all the possible positions of the patients with respect to available healthcare services based on optimum and critical response times.

Conclusion: Locations of public health services in Bandar Abbas were not related to the sites of populations. The RT accessibility technique provides a reasonably sensitive and robust evaluation of accessibility.

Keywords: Health Services Accessibility, Spatial Analysis, Geographic Information System (GIS), Response Time (RT) Accessibility Technique, Bandar Abbas

Copyright: @ 2015 by Kerman University of Medical Sciences

Citation: Masoodi M, Rahimzadeh M. Measuring access to urban health services using Geographical Information System (GIS): a case study of health service management in Bandar Abbas, Iran. Int J Health Policy Manag. 2015;4(7):439-445. doi:10.15171/ijhpm.2015.23

\section{Article History:}

Received: 19 September 2014

Accepted: 31 January 2015

ePublished: 6 February 2015

\section{Key Messages}

Implications for policy makers

- It is possible for the planner to easily recognize which parts of the city have or do not have access to hospitals and clinics during pre-defined response times.

- Hospital(s) and/or clinic(s) with maximum/minimum accessibility can be defined. So, the planners can locate the best places for new health facilities.

- It is possible to compute a summary measure of various types of accessibility between hypothetical patients and health services, especially during the planning stage.

- Response Time (RT) accessibility technique presents detailed information about all the possible positions of the patients with respect to available healthcare services based on optimum and critical response times and vice versa. This technique is important for locating the Emergency Medical Services (EMS).

Implications for public

Patients sometimes have to refer to various health services to receive specific treatments or, the required treatments may not be available in neighboring health facilities. So, comprehensive planning needs to evaluate accessibility of all health services. The result can be presented as brochures or simplified maps. In emergencies, people who live in different parts of the city can use these brochures or maps for selecting the hospitals or clinics.

\section{Introduction}

Accessibility can be judged in both socio-organizational and geographical terms (1). Over the past two decades, an increasing number of health studies together with development of Geographic Information Systems (GISs) technology with transportation modules have integrated the geographical accessibility of services and facilities as an important dimension of the built urban environment (2). Geographical accessibility refers here to the ease with which patients of a given area can reach health services and facilities (3). Early applications of GIS in the health field focused on epidemiological issues (4-6) as the distribution and determinants of health and disease in groups (7). More recently increasing attention on GIS has been paid for public 
health management $(8-11)$ to examine geographical and spatial patterns of health services together with planning the location of new health facilities (12) and also in the spatial analysis of healthcare utilization (13).

Talen has described a number of approaches to measure geographical accessibility (14). Most common approaches are based on distance or travel time to a resource (15). Four types of distance are typically used for calculating accessibility measures: Euclidean distance (straight-line), Manhattan distance (distance along two sides of a right-angled triangle opposed to the hypotenuse), shortest network path distance and shortest network time $(16,17)$. Using different distance types results in discrepancies in geographical accessibility of selected healthcare services (2). Some studies have compared discrepancies in results when geographical accessibility was measured by using different types of distances (2,17-19).

This study investigates geographical accessibility of residential areas in Bandar Abbas (Figure 1a) - an elongated shape city in Hormozgan province on the southern coast of Iran - to healthcare services using different distances. The Floating Catchment Area (FCA) method (20), minimum distance method (14) and Response Time (RT) accessibility technique have been discussed. The main concern is to illustrate the extent of the intra-urban variation in accessibility to general health services, and capability of each method for measuring accessibility and correlation between different types of distances in Bandar Abbas city. There is not acknowledge about the usual place of daily activity and/or inter-urban daily population migration.

\section{Methods}

Bandar Abbas is a coastal city with a population of 588,288 in 2011 (Statistical Center of Iran), an area of 10,493 $\mathrm{km}^{2}$, high population growth (3.40\%) (Vice-Presidency for Strategic Planning and Supervision) and a total of 16 medical health services ( 8 hospitals and 8 clinics). The hospitals and clinics information was inventoried from the website of the Hormozgan University of Medical Sciences (HUMS) and via a field visit. Detailed traffic information was provided by the Central Police Department of Bandar Abbas. We used average driving speed - reported by Central Police Department - for calculations but it can be designed for different times during a day, or even different day during a week can be noted.

This study involves the use of standard GIS functionality such as buffering (e.g. generating catchments at physical distances away from health services), overlay analysis (e.g. examining the location of patients in relation to such areas), network analysis (using characteristics of a network such as traffic flow to gauge how long it takes patients to access a facility), querying classes and creating several different kinds of graphs. In the following, we discuss the procedures.

\section{Data gathering and map preparation}

Interurban road-map, traffic flow data together with hospital/ clinic information (name and location) were inputted into GIS (Figure 1a).

\section{Locating the Geographic Centroids (GC)}

In advanced urban planning, the designer usually predicts some hypothetical stations for various targets such as health

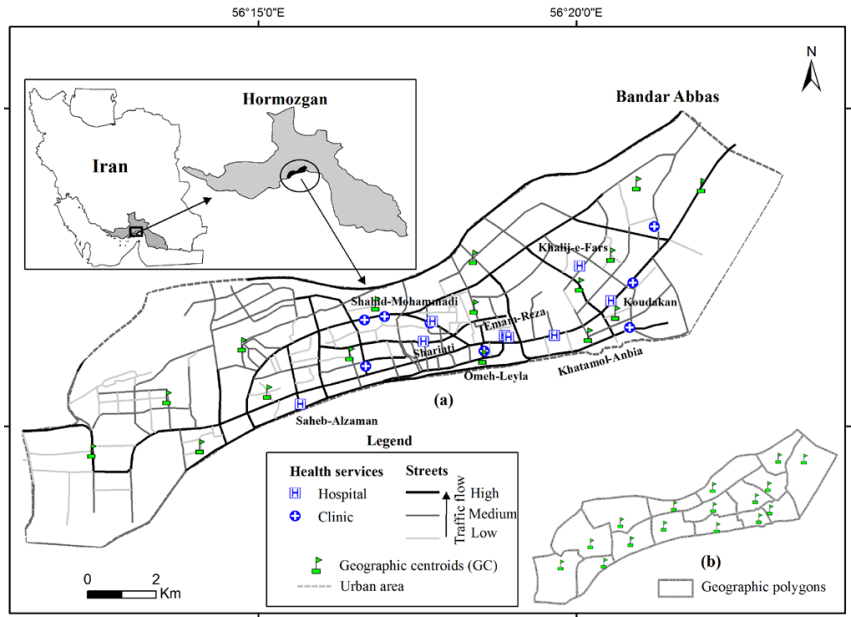

Figure 1. Bandar Abbas, Iran with health services and main population centers as Geographic Centroids (GC) (a) and geographic polygons (b).

services. Local populations with relational databases have allowed managers and planners of health and social services to use GIS to better run their services (13). In the absence of detailed locational data for individuals accessing health services, as in the case of Bandar Abbas, population demand is usually summarized at the population-weighted or, more commonly, Geographic Centroids (GC) of such areas (21). Pre-defined stations considered here as patient's locations referred as the GC (Figure 1). In the absence of individual household locations and population counts, remote sensing technology with high-resolution satellite images, i.e. QuickBird with $60 \mathrm{~cm}$ resolution, were used for calculating the number of homes located inside a geographical polygon. Due to the lack of census tracts in Bandar Abbas, the centroids of each geographical polygon containing at least 500 buildings were denoted as GC by using GIS (Figure 1b). We know that summarizing a population of a zone by assuming all live at the centroid may introduce errors in estimation of accessibility (3). Considering this, the points that we have selected as GC in each polygon, have equal traffic accessibility with respect to most of their polygon's boundary. So, we have tried to minimize the errors. Sixteen GC were located in the territory of Bandar Abbas (Figure 1).

\section{Creating buffer zones}

"Buffer" is an area of specified width drawn around one or more map elements (22). In order to calculate health servicesto-GC ratio for each GC, the FCA method (20) uses circles of varying radii with straight-line distances (to buffer an arbitrary Euclidean distance based on density of healthcare services) placed at the centroids of geographic polygons (GC) and counts the number of health services within the circles. This method is referred as the coverage method by some authors (14). There are also questions regarding the sensitivity of the health services-to-GC ratios to the size of the radius of the circle used in the floating catchment methodology. In Bandar Abbas, three buffer zones with 500, 1,000 and 2,000m widths were drawn separately around the hospitals. Considering the overlaps of the varying buffer zones, a width of $500 \mathrm{~m}$ was specified as the optimal radius. Six buffer zones were drawn for hospitals (Figure 2a). Since clinics have lower facilities for 


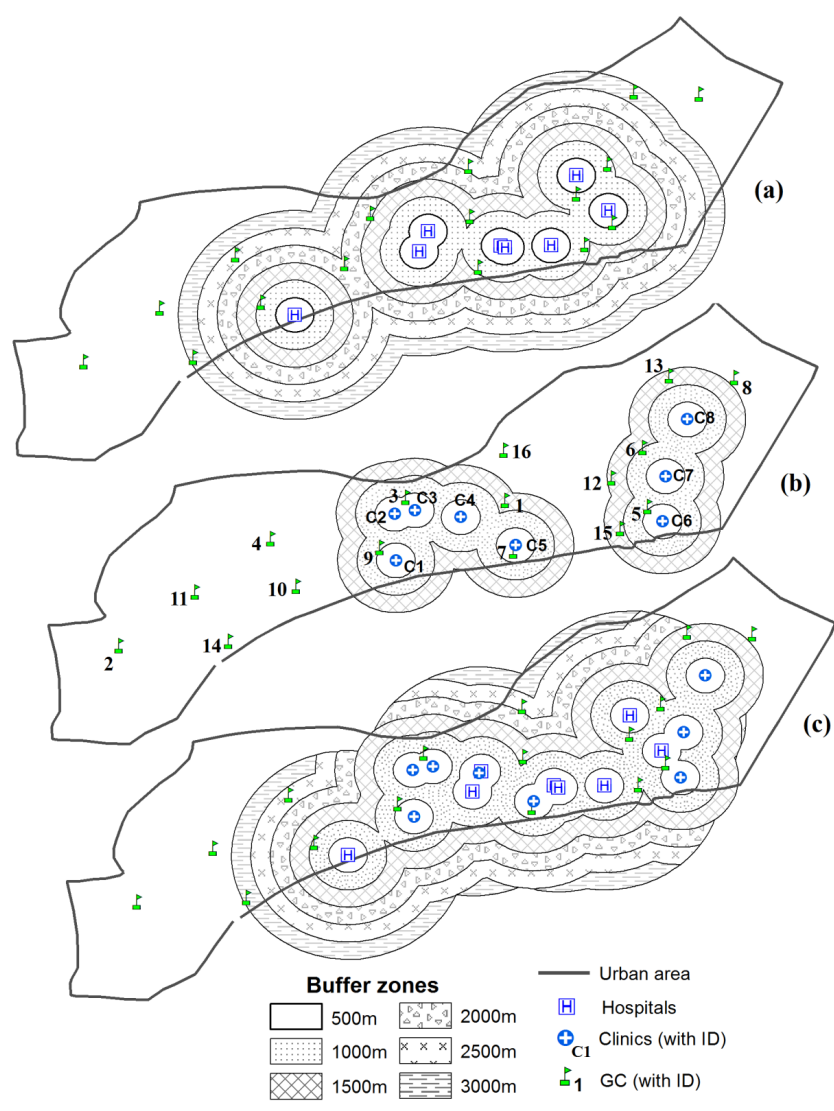

Figure 2. Five hundred meters buffer zones centered on hospitals (a), clinics (b) and hospitals together with clinics (c). Clinics and GC locations presented in (b).

patients, three buffer zones were drawn for them (Figure $2 b$ ). For hybrid state (hospitals and clinics) six buffer zones were drawn (Figure 2c).

\section{Calculation of different types of distances between Geographic Centroids (hypothetical patients) and healthcare services}

Different types of distances were calculated between healthcare services (hospitals and clinics) and patients (GC) (Figures 3, 4 and 5). The results were used for evaluation of the minimum distance method. Usually, patients refer to neighboring hospitals or clinics. In some cases patients need special care and they have to be referred to another hospital or clinic. Hence offering an obvious and decision-making plan for patients to select hospitals/clinics is necessary.

Using Response Time (RT) as a boundary for evaluation of accessibility

RT here means the time that it takes for emergency responders to arrive at the scene of the patient. There is no official standard for response times. RT standards frequently do exist in the form of contractual obligations between communities and Emergency Medical Services (EMS) provider organizations, however. As a result, there is typically considerable variation between standards in one community and another. EMS agencies are increasingly being held to an ambulance RT criterion of responding to a medical emergency within 8 minutes for at least $90.0 \%$ of calls. Berkeley city mandates 10 minutes, or 8 minutes RT (23). While some communities have moved RT standards to $12-15$ minutes (24). It is generally
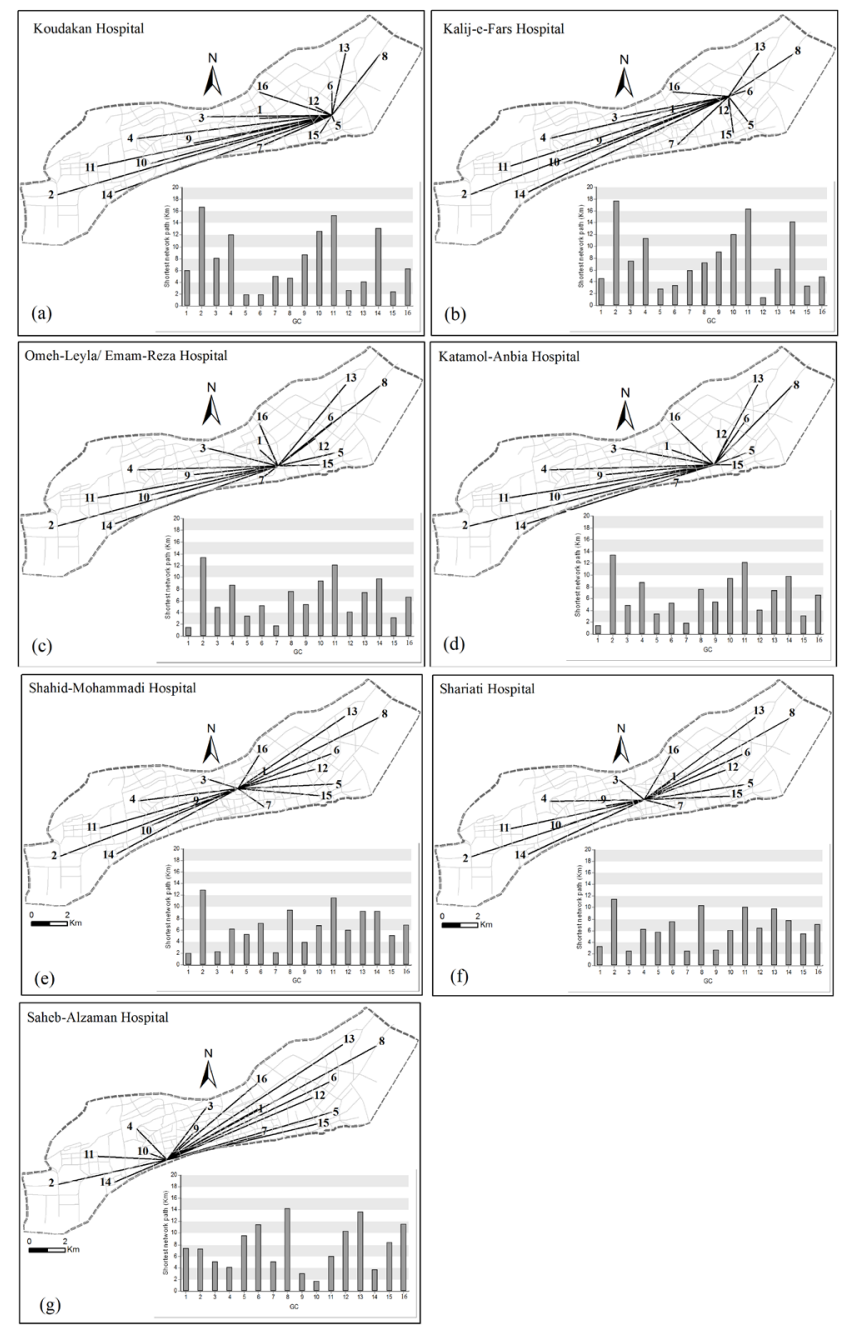

Figure 3. Euclidean distances between GC and hospitals were presented on maps. Graphs show shortest network path distances between GC and hospitals. For Hospitals locations see Figure 1.

accepted within the field that an 'ideal' RT for emergency calls would be between 8 to 15 minutes. With having the accurate shortest network time, we used 8 and 15 minutes RT as optimum and critical boundaries for evaluation of accessibility between health services and GC, respectively.

Results

Floating Catchment Area (FCA) method

In the FCA method, for conceptualizing and measuring the geographical accessibility of health services, the number of health services within each buffer zone were counted (Figure 2a) (20). The accessibility categorized as: excellent $(>1,000$ $\mathrm{m})$; $\operatorname{good}(1,000-2,000 \mathrm{~m})$; fair $(2,000-3,000 \mathrm{~m})$; and poor $(>3,000 \mathrm{~m})$. The results (Figure $2 \mathrm{a})$ indicated that $44.00 \%$ of hospitals had accessibility less than 1,000m to GC (GC 1, $5,6,7,10,12$ and 15), 18.00\% of hospitals had accessibility between 1,000 to $2,000 \mathrm{~m}$ to GC (GC 3, 9 and 16), $12.00 \%$ of hospitals had accessibility between 2,000 to $3,000 \mathrm{~m}$ to GC (GC 3 and 14) and $25.00 \%$ of hospitals had accessibility more than $3,000 \mathrm{~m}$ to GC (GC 2, 8, 11, and 14).

In developing cities with low to medium facilities as Bandar Abbas, clinics play an important role in healthcare. Clinics provide some medical care and support regions with poor 


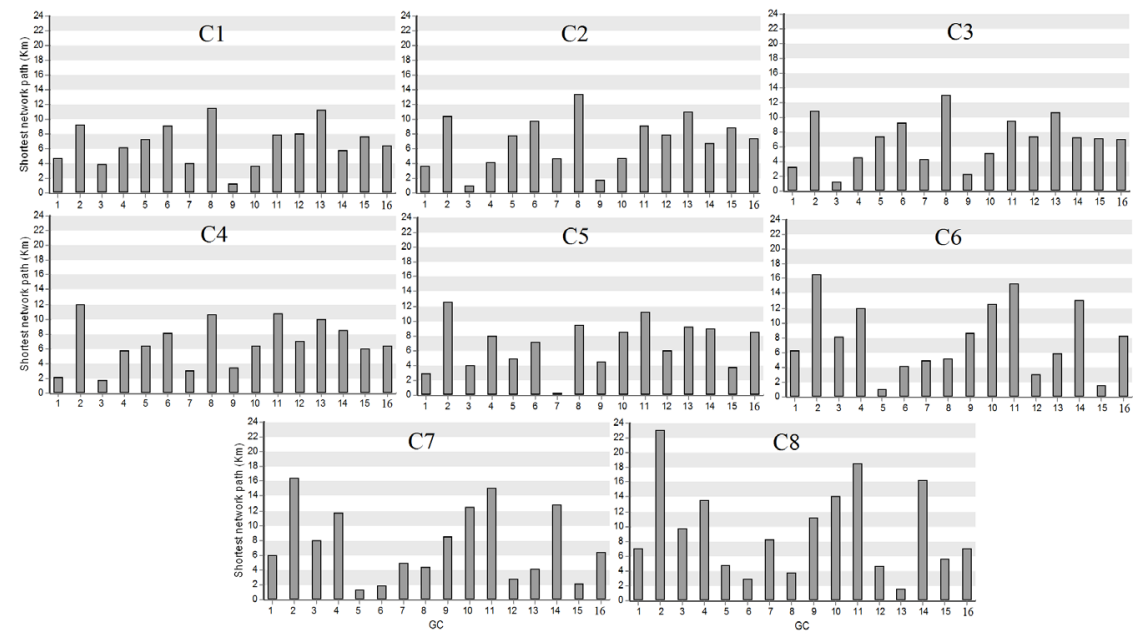

Figure 4. Shortest network path distances between GC and clinics. For clinics locations see Figure $2 \mathrm{~b}$.
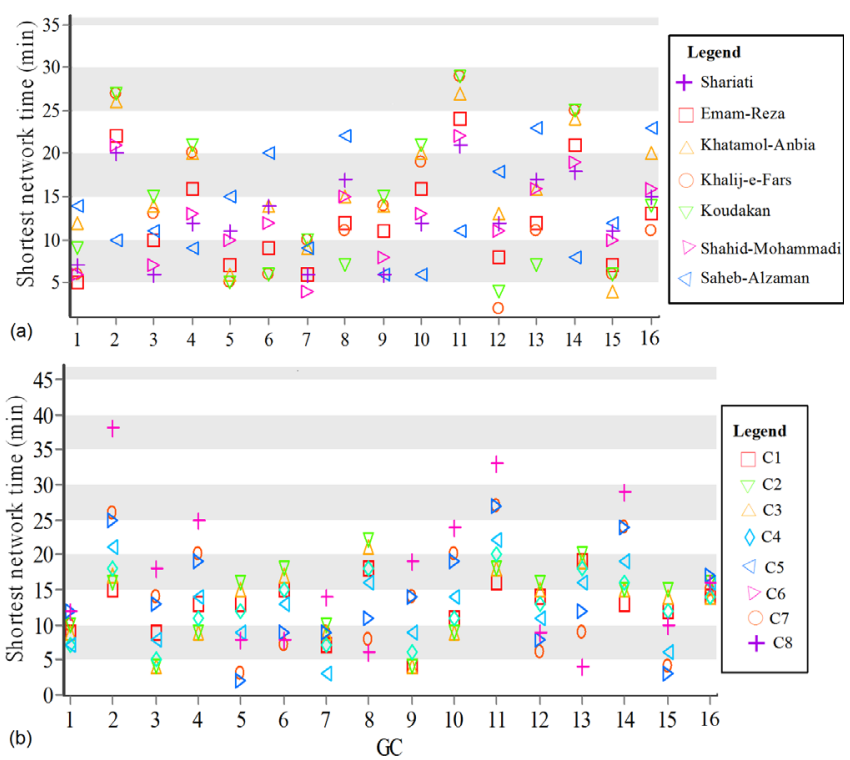

Figure 5. Shortest network time distances between GC and hospitals (a)/clinics (b).

access to hospitals. So, their distribution and accessibility must be considered. Accessibility of hypothetical patients (GC) to clinics in Bandar Abbas also indicated that (Figure 2b) $25.00 \%$ of clinics had accessibility less than $500 \mathrm{~m}$ to GC (GC 3, 5, 7 and 9), 31.00\% of clinics had accessibility between 500 to $1,500 \mathrm{~m}$ to GC (GC 1, 6, 12, 13 and 15) and $44.00 \%$ of clinics had accessibility more than $1,500 \mathrm{~m}$ to GC (GC 2, 4, 8, $10,11,14$, and 16).

Evaluation of accessibility to the hospitals together with clinics in Bandar Abbas (Figure 2c) indicated that clinics did not improve accessibility of those regions with more than 3,000m to GC (GC 2, 8, 11 and 14 in Figures 2a and 2c).

\section{Minimum distance method}

Euclidean distance (straight-line) between hospitals and GC have been presented in Figure 3. This method is referred as the minimum distance method (the distance between a point of origin and the nearest facility) (14).

\section{Response Time (RT) accessibility technique}

We used a new procedure for declaring accessibility to healthcare. Optimum and critical response times (8 and 15 minutes, respectively) have been used for evaluation of accessibility. This technique is named here as "RT accessibility". Changes of the official standards for RT can be easily modified in this method. Based on our new data (Figures 3, 4 and 5), accessibility to the health services during 8 and 15 minutes RT has been evaluated (Table 1).

On the other hand, evaluation of accessibility of every GC with respect to hospitals/clinics during 8 and 15 minutes RT has been presented (Figures 6a, 6b, 6c and 6d).

\section{Discussion}

A major assumption in many of the previous studies is that patients will use their nearest health facility. However, some studies have found that this may not necessarily be the case (25). The FCA method evaluates the accessibility of patients with respect to neighboring health services and utilizes only straight-line or Euclidian distance criterion. But when modelling the accessibility, every member of the population is a potential user of the health services (2) and they use network distance for accessing these facilities. Patients sometimes have to refer to various health services due to specific treatments or, the required treatments may not be available in neighboring health service. So, the pattern of spatial accessibility will depend on the relative location of the population and services $(1,26)$. Some studies have examined the spatial relationship between the location of potential patients and detailed patterns of health service utilization (27). So, comprehensive planning in developing cities with low to medium facilities needs to evaluate accessibility of all of the health services (hospitals and clinics) with respect to every GC as we have done in this study (Figures 3, 4 and 5). The FCA method (Figure 2) indicated that: 1) Of the 8 hospitals in Bandar Abbas, 7 (88.00\%) are located in less than $30.00 \%$ of the urban area; 2) Optimum and maximum accessibility of hospitals to the urban area are 44.00 (accessibility less than $1,000 \mathrm{~m}$ ) and $62.00 \%$ (accessibility less than $2,000 \mathrm{~m}$ ), respectively; 3 ) 
Table 1. Accessibility of hospitals and clinics to GC during 8 and 15 minutes RT.

\begin{tabular}{lll}
\hline Health services & $\mathbf{8}$ minutes accessibility & 15 minutes accessibility \\
\hline Koudakan hospital & $25.00 \%(5,6,12,15)^{*}$ & $56.25 \%(1,5,6,7,8,12,13,15,16)$ \\
Khalij-e-Fars hospital & $25.00 \%(5,6,12,15)$ & $62.50 \%(1,3,5,6,7,8,12,13,15,16)$ \\
Emam-Reza hospital & $25.00 \%(1,5,7,15)$ & $62.50 \%(1,3,5,6,7,9,12,13,15,16)$ \\
\hline Khatamol-Anbia hospital & $12.50 \%(5,15)$ & $43.75 \%(1,5,6,7,12,13,15)$ \\
Shahid-Mohammadi hospital & $25.00 \%(1,3,7,9)$ & $68.75 \%(1,3,4,5,6,7,9,10,12,15,16)$ \\
Shariati hospital & $25.00 \%(1,3,7,9)$ & $62.50 \%(1,3,4,5,7,9,10,12,15,16)$ \\
Saheb-alzaman hospital & $18.75 \%(9,10,14)$ & $56.25 \%(1,2,3,4,7,9,10,11,14)$ \\
C1 & $25.00 \%(3,7,9,10)$ & $56.25 \%(1,3,4,5,7,9,10,14,16)$ \\
C2 & $18.75 \%(1,3,9)$ & $50.00 \%(1,3,4,7,9,10,14,16)$ \\
C3 & $18.75 \%(1,4,9)$ & $68.75 \%(1,3,4,5,7,9,10,12,14,15,16)$ \\
C4 & $25.00 \%(1,4,7,9)$ & $62.50 \%(1,3,4,5,7,9,10,12,15,16)$ \\
C5 & $25.00 \%(1,3,7,15)$ & $50.00 \%(1,3,5,6,7,9,12,15)$ \\
C6 & $18.75 \%(5,12,15)$ & $50.00 \%(1,5,6,7,8,12,13,15)$ \\
C7 & $25.00 \%(5,6,12,15)$ & $56.25 \%(1,5,6,7,8,12,13,15,16)$ \\
C8 & $18.75 \%(6,8,13)$ & $50.00 \%(1,5,6,8,12,13,15,16)$ \\
\hline
\end{tabular}

Emam-Reza and Omeh-Leyla hospitals were considered as a single hospital because they are very close to each other. For hospitals information see Figure $1 \mathrm{a}$ and for clinics and GC location see Figure $2 \mathrm{~b}$.

*Koudakan hospital provided accessibility to $25.0 \%$ of GC including GC 5, 6, 12 and 15 during 8 minutes Response Time (RT).
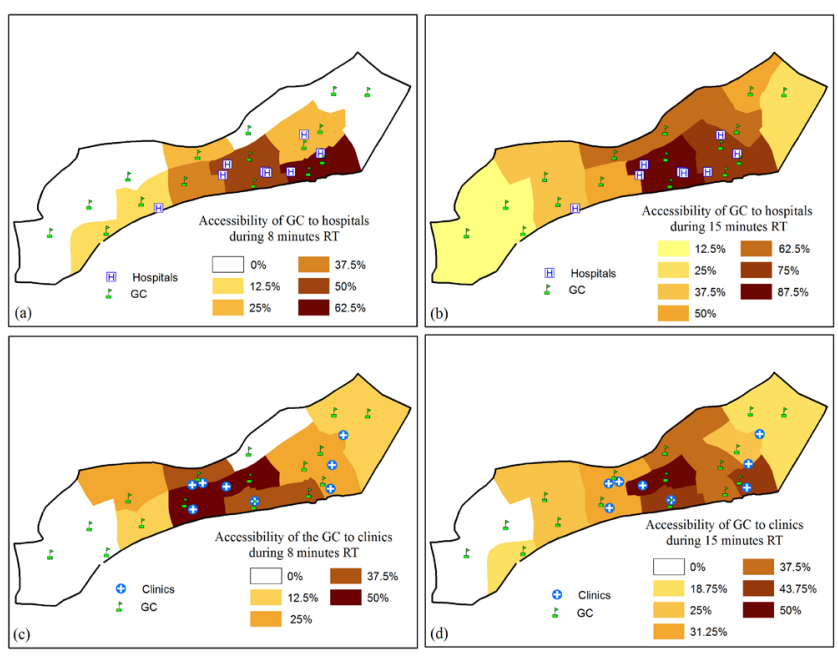

Figure 6. Accessibility of GC to hospitals during 8 minutes (a) and 15 minutes (b). Accessibility of GC to clinics during 8 minutes (c) and 15 minutes $(d)$.

$35.00 \%$ of urban area was covered by clinics; 4) Maximum accessibility of clinics to the urban area is $56.00 \%$ (accessibility less than 1,500m); and 5) Clinics did not improve accessibility to the poor GC (accessibility more than 3,000m). Locations of general health services in Bandar Abbas are not related to the sites of populations or to the hypothetical patients. This small area contains about $88.00 \%$ of the city's hospitals and $35.00 \%$ of clinics.

The comparison of different types of distances between hospital and GC in the Bandar Abbas demonstrated that shortest network time was strongly correlated with shortest network path across the urban area (Pearson correlation greater than 0.91 ) (Table 2) and was moderately correlated with Euclidean distance (Table 2). For clinics, this comparison demonstrated that shortest network time was also strongly correlated with shortest network path (Pearson correlation greater than 0.98) and good correlation was observed for Euclidean distance (Table 3). Unlike the previous results in North American cities $(2,17)$, more accurate measures of distance (shortest network path and time distances) in
Table 2. Global Pearson correlations between alternative types of distance between hospitals and GC in Bandar Abbas.

\begin{tabular}{lccc}
\hline & $\begin{array}{c}\text { Shortest } \\
\text { network path }\end{array}$ & $\begin{array}{c}\text { Shortest } \\
\text { network time }\end{array}$ & $\begin{array}{c}\text { Euclidean } \\
\text { distance }\end{array}$ \\
\hline Shortest network path & 1 & - & - \\
Shortest network time & 0.918 & 1 & - \\
Euclidean distance & 0.597 & 0.375 & 1 \\
\hline
\end{tabular}

$\mathrm{GC}=$ Geographic Centroids

Table 3. Global Pearson correlations between alternative types of distance between clinics and GC in Bandar Abbas.

\begin{tabular}{lccc}
\hline & $\begin{array}{c}\text { Shortest } \\
\text { network path }\end{array}$ & $\begin{array}{c}\text { Shortest } \\
\text { network time }\end{array}$ & $\begin{array}{c}\text { Euclidean } \\
\text { distance }\end{array}$ \\
\hline Shortest network path & 1 & - & - \\
Shortest network time & 0.981 & 1 & - \\
Euclidean distance & 0.912 & 0.844 & 1 \\
\hline
\end{tabular}

$\mathrm{GC}=$ Geographic Centroids

Bandar Abbas -an example of elongated-shaped coastal cities - suggested that Euclidean distances were only moderately correlated with network distances (Tables 2 and 3).

The minimum distance method illustrated that the maximum, median and mean of Euclidean distances between healthcare and GC were $14.20,4.70$ and $5.20 \mathrm{~km}$, respectively. Only $57.50 \%$ of GC had distances lower than mean Euclidean distance.

The RT accessibility (Figure 6 and Table 1) method illuminated that: 1) Five hospitals (25.00\% of hospitals) have provided maximum accessibility to GC during 8 minutes RT and Khatamol-Anbia hospital had minimum coverage (12.50\%); 2) GC 2, 4, 8, 11, 13 and 16 had no access to hospitals during 8 minutes RT (Figure 6a); 3) Shahid-Mohammadi and Khatamol-Anbia hospitals presented maximum (68.70\%) and minimum (43.70\%) accessibility during 15 minutes RT, respectively, with respect to GC; 4) All of the GC had access to hospitals during 15 minutes RT (Figure 6b); 5) Four clinics provided maximum accessibility to GC during 8 minutes RT (25.00\% of clinics) and four other clinics had poor coverage (18.70\%); and 6) C1 had provided maximum accessibility of 
the clinics to GC during 15 minutes RT (68.70\%) and four clinics (C2, C5, C6 and C8) had poor accessibility (50.00\%). By this method, it is possible for the planner to easily recognize which parts of the city have or have not access to hospitals (Figures 6a and b) and clinics (Figures $6 \mathrm{c}$ and d) during pre-defined response times. For example, results of RT accessibility in Figure 6 and Table 1 specified that: in comparison to other GC, GC 5 and 15 that were located in territory of Khatamol-Anbia hospital had maximum accessibility to hospitals during 8 minutes RT. Considering the results of our calculations which indicated that KhatamolAnbia hospital had minimum accessibility (12.50\%) to all GC, running Khatamol-Anbia hospital in its present position has not been a good idea. Saheb-Alzaman hospital also provided poor (18.70\%) accessibility to all GC during 8 and 15 minutes RT. But it is the only hospital that provided accessibility to GC 10 and 14 during 8 minutes and GC 2, 4 and 11 during 15 minutes RT. GC 8, 13 and 16 that had not access to hospitals during 8 minutes RT, improved by Koudakan hospital in 15 minutes RT. C8 clinic also improved accessibility of GC 8 and 13 during 8 minutes RT.

Field queries specified that satisfactory accessibility of practitioners is the only criterion involved in running Khatamol-Anbia hospital and the study results illuminated its poor accessibility to other parts of the urban area. It must be accepted that ensuring adequate accessibility of practitioners is by no means the only criterion involved in running a satisfactory health service.

\section{Conclusion}

This study suggests that the RT accessibility technique provides a better assessment of accessibility as compared to other methods. It presents detailed information about all the possible positions of the patients with respect to available healthcare services based on optimum and critical response times and vice versa. Since this technique utilizes the shortest network path and time distances, then it provides a reasonably sensitive and robust evaluation of the accurate accessibility. Locations of general health services in Bandar Abbas are not related to the sites of populations or to the hypothetical patients. This small area contains about $88 \%$ of the city's hospitals and $35 \%$ of clinics. The results highlight that it is possible to compute a summary measure of various types of accessibility between hypothetical patients and health services, especially during the planning stage.

\section{Acknowledgments}

The authors express the gratitude to the research council of Hormozgan University of Medical Sciences and Hormozgan University, Iran for the financial support. We also thank the Central Police Office of Bandar Abbas for providing detail traffic information.

\section{Ethical issues}

The study was approved by the ethics committee of Hormozgan University of Medical Sciences (HUMS)

\section{Competing interests}

Authors declare that they have no competing interests.

\section{Authors' contributions}

MM and MR both developed the concept. MM provided the GIS data and performed the data analysis. MR wrote the initial draft ofmanuscript and both authors contributed to the revisions of the manuscript.

\section{Authors' affiliations}

'Department of Geology, Faculty of Sciences, Hormozgan University, Bandar Abbas, Iran. ${ }^{2}$ Department of Biochemistry, Faculty of Medicine, Hormozgan University of Medical Sciences, Bandar Abbas, Iran. ${ }^{3}$ Food and Cosmetic Health Research Center, Hormozgan University of Medical Sciences, Bandar Abbas, Iran.

\section{References}

1. Joseph AE, Phillips DR. Accessibility and utilization: geographical perspectives on health care delivery. New York: Harper \& Row; 1984.

2. Apparicio P, Abdelmajid M, Riva M, Shearmur R. Comparing alternative approaches to measuring the geographical accessibility of urban health services: Distance types and aggregation-error issues. Int $J$ Health Geogr 2008; 7: 7 . doi: 10.1186/1476-072x-7-7

3. Hewko J, Smoyer-Tomic K E, Hodgson MJ. Measuring neighbourhood spatial accessibility to urban amenities: Does aggregation error matter? Environ Plan A 2002; 34: 1185-206. doi: $10.1068 / a 34171$

4. Glass D. A world health organization pilot study involving environment, public health and GIS. Mapping Awareness and GIS in Europe 1991; 6: 36-40.

5. Nicol J. Geographic information systems within the national health service: the scope for implementation. Planning Outlook 1991; 34: 37-42. doi: 10.1080/00320719108711887

6. Dunn C. GIS and Epidemiology. Education, Training and Research. London: Association of Geographic Information; 1992.

7. Sackett DL, Haynes RB, Guyatt GH, Tugwell P. Clinical Epidemiology. A Basic Science for Clinical Medicine. London: Little, Brown and Co Inc.; 1991.

8. Thrall GI. The Future of GIS in Public Health Management and Practice. J Public Health Manag Pract 1999; 5: 82. doi: 10.1097/00124784-199907000-00014

9. Roper WL, Mays GP. GIS and Public Health Policy: A New Frontier for Improving Community Health. J Public Health Manag Pract 1999; 5: vi-vii. doi: 10.1097/00124784-199905020-00002

10. Kulldorff M. Geographic Information Systems (GIS) and Community Health: Some Statistical Issues. J Public Health Manag Pract 1999; 5: 100-6. doi: 10.1097/00124784-19990502000038

11. Yasnoff WA, Sondik EJ. Geographic Information Systems (GIS) in public health practice in the new millennium. J Public Health Manag Pract 1999; 5: ix-xii. doi: 10.1097/00124784-19990700000002

12. Dowie PJ, Koval SJ, Burnhill PM, Healy RG. GIS and community health care: a study of geriatric services provision; 1995.

13. Foley R. Assessing the applicability of GIS in a health and social care setting: planning services for informal carers in East Sussex, England. Soc Sci Med 2002; 55: 79-96. doi: 10.1016/ s0277-9536(01)00208-8

14. Talen E. Neighborhoods as service providers: a methodology for evaluating pedestrian access. Environ Plann B Plann Des 2003; 30: 181-200. doi: 10.1068/b12977

15. Talen E, Anselin L. Assessing spatial equity: an evaluation of measures of accessibility to public playgrounds. Environ Plan A 1998; 30: 595-613. doi: 10.1068/a300595

16. Fotheringham AS, Brunsdon C, Charlton M. Quantitative geography: Perspectives on spatial data analysis. London: Sage Publications; 2000.

17. Apparicio P, Brochu M, Dussault G. The measure of distance in a social science policy context: Advantages and costs of using network distances in eight Canadian metropolitan areas. Journal of Geographic Information and Decision Analysis 2003; 7: 10531.

18. Brabyn L, Skelly C. Modeling population access to New Zealand public hospitals. Int J Health Geogr 2002; 1: 3.

19. Fone DL, Christie S, Lester N. Comparison of perceived and modelled geographical access to accident and emergency 
departments: a cross-sectional analysis from the Caerphilly Health and Social Needs Study. Int J Health Geogr 2006; 5: 16.

20. Luo W. Using a GIS-based floating catchment method to assess areas with shortage of physicians. Health Place 2004; 10: 1-11. doi: 10.1016/s1353-8292(02)00067-9

21. Higgs G. A literature review of the use of GIS-based measures of access to health care services. Health Serv Outcomes Res Methodol 2004; 5: 119-39. doi: 10.1007/s10742-005-4304-7

22. Aronoff S. Geographic Information Systems: A Management Perspective. Ottowa: WDL Publications; 1991.

23. Hogan AM. Audit: Response times to calls for emergency medical services could be improved. Berkeley: Office of the City Auditor; 2008
24. Ludwig GG. EMS Response Time Standards [internet]. 2004 Available from: http://www.emsworld.com/article/10324786/ ems-response-time-standards

25. Martin D, Williams $\mathrm{H}$. Market-area analysis and accessibility to primary health-care centres. Environ Plan A 1992; 24: 1009-19. doi: 10.1068/a241009

26. Joseph AE, Bantock PR. Measuring potential physical accessibility to general practitioners in rural areas: a method and case study. Soc Sci Med 1982; 16: 85-90. doi: 10.1016/02779536(82)90428-2

27. McLafferty SL. GIS and health care. Annu Rev Public Health 2003; 24: 25-42. doi: 10.1146/annurev.publhealth.24.012902.141012 